\title{
Artificial, augmented and automated chemistry
}

\author{
Jeremy Frey, professor of physical chemistry at the University of Southampton and principal investigator for the \\ AI3SD Network+, talks with Nature Chemistry about the perils of uncertainty in the quality of machine learning data
} and the synergies between $\mathrm{Al}$ and other technologies.

You are the principal investigator for the AI3SD Network+. Can you explain what the AI3SD Network+ is?

The AI3SD stands for Artificial Intelligence, Augmented Intelligence, for Automated Investigation for Scientific Discovery, which is a bit of a mouthful! We are a UK Research and Innovation Engineering and Physical Sciences Research Council (UKRI EPSRC) Network+. As a Network ${ }^{+}$we are funded to organize a range of activities. As well as running events and workshops, we have funded several small feasibility projects, attendances at conferences, summer internship programmes, and many other activities (see our website www.ai3sd.org). Hands-on events such as hackathons have been very successful, but COVID-19 meant that we needed to shift all events online. This actually led to a doubling of the size of the Network!

The success of the Network is due to my co-investigator Mahesan Niranjan, our very effective network coordinator Samantha Kanza, and our active and supportive advisory board chaired by Prof. John Overington. At our first advisory board, it was pointed out that we should have added one more 'AI' and then we could have had the acronym AI4ScienceDiscovery. Unfortunately, the address ai4science.org was already taken (though it seems by another member of our advisory board who had forgotten about this) but we do plan to use ai4science.network in the future. However, we have been debating exactly what the 4 th AI should be!

What is the Network trying to achieve? And what areas of chemistry have you chosen to focus on?

The call from EPSRC asked for a network that would bring cutting-edge science together with cutting-edge AI computing. Though reasonable funding was available, it was certainly not enough to cover all of science, so our application focussed on the related fields of chemistry and materials. Our aim was to bring together researchers involved at the forefront of AI - both traditional knowledge engineering aspects and the more recent incarnations of machine learning - and those involved in experimental and theoretical chemistry. We

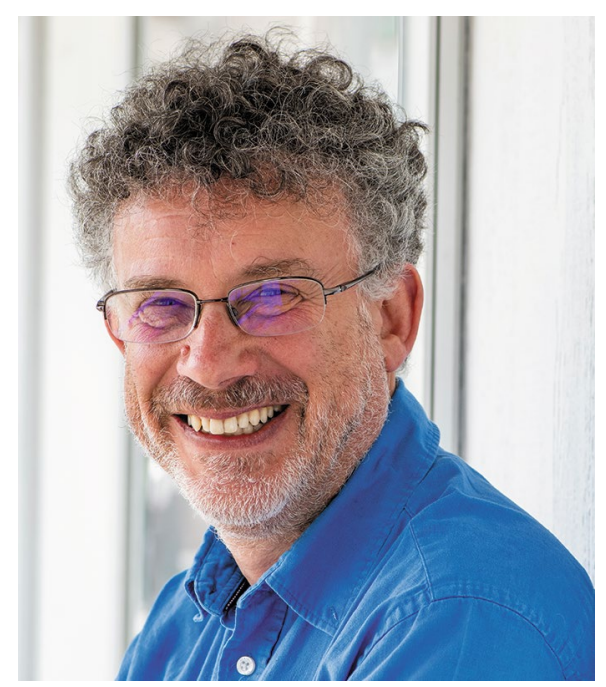

Credit: University of Southampton.

have tried to keep a broad view of chemistry. Many of the projects we funded have come from drug discovery or materials design and have engaged chemists, computer scientists and mathematicians. In our workshop themes we have ensured a broader view of molecular and materials properties.

Because scientific discovery is the focus of this Network, we want to go beyond simply spotting correlated patterns, as is often done in the use of machine learning methods. We question what is meant by conventional discovery and what it will mean in the future when assisted by AI, hence we have Will McNeill, a philosopher of science as part of the consortium.

Personally, the Network has been a fantastic opportunity to meet, appreciate and learn from the experience and research of colleagues in computer science and chemistry, in academia, industry and government, nationally and internationally.

What makes these areas of science
well suited for artificial intelligence and
machine learning approaches? And are
there any areas or aspects of chemistry
that you think are not suited to these
approaches?
One consistent theme that has emerged
from all our workshops, talks and

discussions is the need for data; there is rarely enough data and the data that is available is often of uncertain quality (poor quality is not as much of a problem as uncertain quality). So, areas where data is available, for example using high throughput synthesis and characterization, are clear areas for application of machine learning and potentially can support even the deep learning machine learning (ML) approaches. However, in very few cases is there any chance of applying the types of deep learning seen in the application of AI/ ML to games where computer-generated data is available (that is, a programme plays against itself).

Issues of available computer power arise for the high-level quantum mechanics/ quantum chemistry (QM/QC) calculations on both the training and test datasets. Such obstacles limit even applications of AI/ML to quantum chemistry where $\mathrm{ML}$ is used to improve the accuracy of lower-level QM calculations. Similar considerations apply to molecular dynamics simulations where ML techniques have been used to accelerate the integration.

\section{Artificial intelligence often requires a lot of data, yet there is usually an error associated with measuring the results of chemical experiments such as reaction yields or rates, and often a limit to the precision with which these properties can be measured. How can the community resolve this? And do you see this mainly as an experimental or software problem?}

As noted in my answer to the previous question, data is critical. Current ML approaches certainly need lots of high-quality data (ML approaches make use of poor data when the uncertainty is understood, filtering out poor quality data is also possible using ML approaches but then that limits the breadth of data available). In principle, enough data is available for many chemical properties, as long as we have high-throughput techniques for both synthesis and characterization (and don't need much of the substance itself). Obtaining quality data is much more difficult for more variable data, such as reaction yield, which can depend critically 
on conditions, so there is a much larger experimental space to cover. When it comes to biological response then there is even greater variability, and thus difficulty. As an experimental issue, we need to be able to work at scale and with increased reliability - so there are both hardware and software/data issues.

A related way of looking at this is that unlike in classic pattern recognition systems - such as computer vision for self-driving cars, for which gathering vast quantities of data is feasible and desirable - scientific discovery as a problem domain comes with extensive prior knowledge, captured in more classic physical models. The challenge in the not-so-large data regime is to exploit such knowledge in combination with data-driven models. We have looked at many such methodologies in our workshops.

What aspects of artificial intelligence do you think are most misunderstood by the wider scientific community? And what can be done to address this?

What can AI actually achieve? Realistic expectations of what can be done in predicting molecular properties are probably moderately in line with the wider community view; however, the prediction of the properties of complex formulations, such as those used in drug delivery, materials coatings, functional materials and biological interactions, are still limited compared to what many in the wider community imagine is possible. Even those who recognize that the limitations of the available data prevent applications of ML probably think that if only we have plenty of data, then the sky is the limit (yes, including atmospheric and environmental chemistry). The reality is that the heterogeneous nature and complexity of chemistry still outstrips computational power. A combination of ML and computational chemistry and new insight is required.

The issues of bias are present in AI/ ML applications to chemistry. Perhaps not as immediately serious as some other areas. Unintended bias due to selection of the training set not picked up via the test set, is well known but is easily forgotten. This is closely aligned with understanding diversity of chemistry and prompts much work on similar/dissimilar compound sets. Broadening the view here, the application for example to drug design can fall into the same trap, of how the training set influences the possible outcomes, and how appropriate is the training set to different parts of the global community. In the limit, how do we deal with personalized medicine, a ML model for each of us?

\section{Artificial intelligence-based tech- nologies can be powerful approaches but sometimes the field and achievements have been overhyped? Do you have any advice for non-specialists trying to evalu- ate whether claims go beyond what the data and results support? Or are there any common issues that non-specialists should watch out for in chemistry papers?}

Always look carefully at the chemistry. What data was used? What do the molecules or materials look like? How different are the outputs of the model from the inputs? Do the suggested molecules really answer the problem? Are they potential drugs or tested drugs? Suggested materials or materials tested in devices? Is the input data provided and the model explained? These are the areas of unintended bias.

The Network has focused on the crucial question of 'why has the model given these predictions?', do the suggested drugs or materials shine a light on new ideas in or for chemistry? Being able to explain the models is really important for $\mathrm{AI} / \mathrm{ML}$ to make a difference to the understanding of chemistry. These issues also play to the philosophy angle of the Network in thinking about how AI will change the nature of chemical discovery.

Are there any reporting standards for methods, data or custom software that you would like the community to adopt to facilitate the reuse and reproduction of work?

The standards for data; they are far from complete or agreed but things are getting better. The community has a long way to go on standards for describing the ML models. We do have the overall FAIR principles, which do apply equally to data and all aspects of work. Tools such as Jupyter notebooks and the general ideas of literate programming very much apply to ML work in chemistry. In general, I support the use of as many open-source and open-science tools as possible but fully acknowledge the growing range of quality commercial software. Even when using commercial codes, it is essential to be able to describe how the data has been manipulated and to ensure that data models can be exchanged using open formats.

These ideas also go hand-in-hand with the ability to explain ML models. The thrust of the Network is in scientific discovery, but just obtaining a solution to a problem - for example, the next drug, the next material to make, how to optimize a design - is only part of the journey of discovery. The 'why' needs to be understood. Why has the ML system arrived at this answer? Is this an example of bias? Can we account for and explain the result (even in hindsight)? What have we learnt? Can we extract new theory and ideas from these models? That is clearly essential for discovery (the ML becomes part of the experiment).

We can look further to the future and ask if the $\mathrm{AI} / \mathrm{ML}$ approach can not only provide a result and provide a pathway to understanding the 'why' but also begin to aid the asking of the 'what'. Can the augmented intelligence systems aid in proposing the hypothesis as well? There are areas of complex chemistry, such as functional materials and interactions with biology, where the system complexity is such that AI could make a significant impact in this way.

\section{Artificial-intelligence-based} technologies have some clear synergies with robotics and automated detection and analysis. What bottlenecks do you think are currently limiting development into more-autonomous integrated systems?

The synergies between AI and robotics are very interesting. In the wider community, these ideas are probably most clearly linked in autonomous cars/driving and robotic surgery. In chemistry, laboratory robotics have significant potential, mainly, I suspect, in industrial labs at the moment (due to cost), but as we see the adoption of robotics on an almost consumer scale, we can expect to see many more robots in labs. There are now a number of dark labs, effectively fully automated, that are available to researchers - of course contract synthesis and measurements have been available for a long time, and for the user it perhaps does not matter if that lab is robot- or human-run, though the costs and throughput may vary.

I think the complexity of many chemical processes is a limitation on the role of robots. Much work has been done on simplifying processes and standardizing synthesis, making use of flow techniques for example, and in these areas the use of robotics is already advanced. Analogies with biochemical work (I am thinking here of automated DNA, RNA synthesis) show the way when the processes are amenable. Perhaps at the other extreme, bespoke physical chemical experiments benefit from automation and computer control in a different but still very significant manner, and this has driven the experiments for many years. Nevertheless, the rise in AI technologies has the potential to revolutionize the extraction of information (and knowledge) from raw experimental data and thus the nature of what experiments are possible. 
Are there any specific technologies that you'd like to see developed which would expand the usefulness of artificial intelligence and machine learning approaches?

We need to understand how to make predictions based on much smaller amounts of data than much of the recent ML work entails. We might call this small deep learning. This research transcends chemical applications and of course is very important where AI/ML meet human learning. Thinking specifically of chemistry, we need to develop ways of handling energy/ structure landscapes, that is, 3D structure/ conformations as these are critical in the interaction between molecules, whether for complex mixtures, materials or in biological interactions.

A frequent problem in $\mathrm{ML}$ is working with high dimensional spaces, with relatively sparse data, and consequently the need for dimensional reduction. While this is a frequent issue for deep learning, the continued work on algorithms and the mathematical underpinning will be very useful for chemical applications, taking into account the nature of chemical data. A better understanding, quantifying and modelling of 'uncertainty' is going to be useful. As mentioned above with the need for quality data, a limitation of AI systems when we deploy them is of course the 'garbage-in-garbage-out' issue. That is, a trained AI model will always produce an output, even in environments that it is not trained to operate in. Properly quantifying uncertainty and making predictions along with confidence levels in these predictions will often be crucial. Methods founded in
Bayesian inference principles help do this, but scaling them to very large and complex models remains challenging.

Thinking of chemical space, we do need to develop a better understanding of this concept and of course ideally, we still need a map of chemical space! What we can expect such a map to look like is probably the subject of a different discussion and leads to the consideration of chemistry as navigation versus discovery. A static concept of a chemical map is never going to reflect the diversity and flexibility of the discipline. Ideas of molecular or material structure implicitly deal with time and energy and depend on the environment of the molecule or what it is used for. Transformations between species are as important, so in many ways what we are looking for is not just a map, or even a map with pathways overlaid, but a true depiction of chemical space-time. It is my hope that, with explainable AI models, we can start to build out regions of the chemical space-time and gain real insight as well as practical applications.

Any technologies that help understand the interaction of chemistry with biology will support better predictions for drugs, agrichemicals and environmentally sustainable materials.

Quantum computers are an example of new technologies that will become able to make a significant impact on chemistry as the number of qubits available is rising rapidly. In my view they will not just be used to do quantum chemical calculations, but to do things like handling the efficient searching of vast chemical spaces. But with much effort invested in programming chemistry on conventional computers, it will be some time before quantum computers are faster at solving chemical problems. Looking to the future, the Network is planning to run some workshops on programming quantum computers for chemistry.

What improvements in digital infrastructure would you like to see to help and support the chemistry community make wider use of artificial intelligence and machine learning technologies?

We certainly need a much more advanced and global infrastructure. Repositories that encourage data with as much metadata (semantic information) as possible. Ideally, metadata should be machine- and human-readable, with the contextual stories, to enable the community to make the best use of the data we have. We have elements of this story but they are far from complete or easy to use.

Cloud-based systems are making even quite bespoke hardware available to meet the contrasting needs for ML and QC, and even quantum computers are available in this way. We need to start to train chemists to use these systems. The redesign of undergraduate chemistry courses is underway, but reskilling opportunities for highly qualified chemists will also be very important. Equally important is bringing the new capabilities of chemistry to the younger audience and hands-on chemistry \& AI outreach is a must.

Interviewed by Russell Johnson

Published online: 16 August 2021 https://doi.org/10.1038/s41557-021-00779-y 